\title{
Encouraging Witting Participation and Performance in Digital Live Art
}

\author{
Jennifer G. Sheridan \\ BigDog Interactive \\ InfoLab21, South Road \\ Lancaster UK \\ +44 1524770062 \\ jennifer@bigdoginteractive.co.uk
}

\author{
Nick Bryan-Kinns \\ Department of Computer Science \\ Queen Mary, University of London \\ Mile End, London UK \\ +442078827845 \\ nickbk@dcs.qmul.ac.uk
}

\author{
Alice Bayliss \\ School of Perf and Cultural Ind. \\ Bretton Hall Campus \\ University of Leeds UK \\ +441133439237 \\ a.bayliss@leeds.ac.uk
}

\begin{abstract}
We describe a framework for characterizing people's behavior with Digital Live Art. Our framework considers people's wittingness, technical skill, and interpretive abilities in relation to the performance frame. Three key categories of behavior with respect to the performance frame are proposed: performing, participating, and spectating. We exemplify the use of our framework by characterizing people's interaction with a DLA - iPoi. This DLA is based on the ancient Maori art form of poi and employs a wireless, peer-to-peer exertion interface. The design goal of $i P o i$ is to draw people into the performance frame and support transitions from audience to participant and on to performer. We reflect on iPoi in a public performance and outline its key design features.
\end{abstract}

\section{Categories and Subject Descriptors}

H.5.1 [Information Interfaces and Presentation]: Multimedia Information Systems; H.5.2 [Information Interfaces and Presentation]: User Interfaces; J.5 [Arts and Humanities]: Performing arts (e.g., dance, music).

\section{General Terms}

Performance, Design, Experimentation, Human Factors, Theory.

\section{Keywords}

Wittingness, unwitting, digital live art, iPoi, poi, Maori art, performance framing, exertion interface, performer, participant, spectator.

\section{INTRODUCTION}

Digital Live Art (DLA) [24] is the intersection of live art, computing and human-computer interaction (HCI). Research has begun to investigate how the methods and theories in the performance arts can be used to understand human-computer interaction in DLA. Our interest is in how we can encourage people to participate in technically-mediated public performances.

(C) Jennifer G. Sheridan, Nick Bryan-Kinns, Alice Bayliss, 2007

Published by the British Computer Society

People and Computers XXI-HCI... but not as we know it:

Proceedings of HCI 2007

Linden J. Ball, M. Angela Sasse, Corina Sas, Thomas C. Ormerod, Alan Dix, Peter Bagnall, and Tom McEwan (Editors)
Evaluating and measuring interaction in public performance is very different to the frameworks and measures used to understand interaction in more 'traditional' forms of HCI. Traditional HCI has focused on understanding interaction in desktop computing applications. This has typically concentrated on task-based computing and designing interfaces which increase efficiency of task execution, for instance. However, as computing moves away from the desktop to mobile and wireless ubiquitous environments, we see a shift to non taskbased uses of computing $[6,18]$ and understanding the needs of users as performers [24]. To better understand these needs HCI requires an understanding of performance framing.

Gregory Bateson first identified the concept of the 'performance frame' in his paper titled A Theory of Play and Fantasy [2]. The performance frame is the cognitive context where the rules of behavior, symbols and their interpretations are bound within a particular activity within its own structure. The concept has been used extensively in many contexts, including understanding face-to-face encounters in the everyday [13]. In this paper, we update earlier descriptions of performance framing in DLA $[5,11,23]$ to include our definition of wittingness.

Wittingness ${ }^{1}$ [24] is an individual's or group of people's knowledge or awareness of the performance frame. It can be used as a device for tempting performative interaction, or the interaction that occurs within and as a result of the performance frame. Wittingness implies that the individual (or group) has accepted by choice or without reluctance to interact (or to not interact), and therefore they have an established knowledge about the performance frame. On the other hand, unwitting implies that the individual or group does not have any established knowledge about the performance frame nor the possible choices confronting them. So where a witting person chooses to interact, an unwitting person does not even know that they can interact. Witting interaction then, is the activity done within the intended performance frame by witting individuals or a group in front of an audience.

Encouraging transitions in witting behavior allows us to, among other things, implicate bystanders in a performance and to tempt them into crossing the boundaries of 'normal' human behavior $[3,23]$. This provides an opportunity to entice spectators into participating in a public performance which can destabilize our notions of reality and create a blurring of fiction and everyday life.

\footnotetext{
${ }^{1}$ The term witting comes from the Middle English witen which akin to Old High German wizzan - to know, and the Latin - to see.
} 
In our research, we are specifically interested in encouraging transitions which focus on the notion of Paidia, or pure play rather than Ludus, or pure games [8]. Ludic games (and many interactive systems and environments) are often rule-bound and introduce the concepts of winning, combat or conflict. Paidia on the other hand, focuses on the often shared, anarchic and spontaneous play found in improvisation. To entice improvisation, we have created an exertion interface [19] called iPoi. An exertion interface is an interface that requires intense physical effort and while it may have a 'low entry fee' [27], it takes a long time to master. Many exertion interfaces focus on remote interfacing and competitive, Ludic play. For example, Breakout for Two [20] is designed for two players to remotely compete against each other in sports match and, NEAT-oGames [12] is a competitive race between remote participants with interactive avatars. iPoi however, focuses on co-located interaction and Paidiac play, like that experienced by children in the artwork 'ensemble' [1]. We use a wireless and peer-topeer embedded computing system which employs continuous, realtime tangible manipulation and physical representation of acceleration data in real space.

In this paper, we begin with a discussion of current research in HCI which discusses the role of technology in public performance and interactivity. We then identify witting behaviors in performance and means to characterize them borrowing from Beeman [4]. We describe our exertion interface, iPoi, and discuss a public performance in which we observed how iPoi encouraged transitions in witting behavior We conclude with a discussion of how and why our exertion interface allows transitions in witting behavior.

\section{PREVIOUS RESEARCH}

Previous HCI research on understanding the role of interactive technology in public performance has typically maintained a strong distinction between performer and audience, and moreover, has not explored the nature of transitions from spectating to performing. We describe some of this research in this section.

Reeves et al. [21] present a framework for understanding the role of technology in public performances by examining the performers' manipulations and their resultant effects. By classifying design strategies into 'secretive', 'expressive', 'magical', and 'suspenseful' they are able to distinguish between, say, the use of a presentation in a corporate environment, and interaction with an installation in a museum. However, the distinction between 'spectator' (one who watches) and 'performer' (one who does) is such that their framework cannot be used to capture how spectators become performers, for instance, by watching other performers interacting with an installation piece. Moreover, Reeves et al.'s definition of 'performer' is too crude as it encompasses all people who interact with a system, regardless of their skill, insight, or virtuosity.

Furthermore, Reeves et al. do not account for people who are in the same space as the performance, but not implicated in it, nor those who are acquiring the necessary skills and understanding to become a performer. Indeed, the framework implicitly assumes that spectators understand the intention of performers and are able to understand the meaning associated with physical actions. For example, consider the video arcade game 'Dance Dance Revolution' [15] in which participants tread on eight large floor mounted buttons in specific sequences in order to interact with a computer playing music. In this situation observers must know the rules and actions associated with interacting with Dance Dance Revolution in order to understand what the visible physical actions of jumping on eight buttons means, and how that relates to the game itself. In Reeves et al.'s framework there is an implicit assumption that spectators know these rules whereas the game is complex enough that we suggest that the rules are probably best learnt by interacting with it.

Benford et al. [5] developed Reeves et al.'s framework further to include the concept of the 'performance frame' (cf. [2]) which embodies the "principles and conventions by which both [performers and spectators] are able to take part in the performance and interpret what is happening'. In doing so, performers are viewed as frame constructors, whilst spectators are viewed as frame interpreters. Furthermore, they distinguish between witting and unwitting [24] spectators - respectively, 'audience' members who were within the performance frame, and 'bystanders' who may observe performers' actions but do not interpret them as performance. This distinction is subtle, and yet we argue that it still does not capture different behaviors that may be exhibited in and around a performance.

By analogy, consider Clark's rich characterization [9] of participants in a conversation illustrated in Figure 1. For Clark the Speaker and Addressee are central to the conversation. Clark also identifies Bystanders with reference to the conversation, and he additionally identifies Side participants and Eavesdroppers. As we shall see in Section 3, a comparably detailed level of analysis is needed to characterize the different levels of wittingness of behaviour in and around a performance.

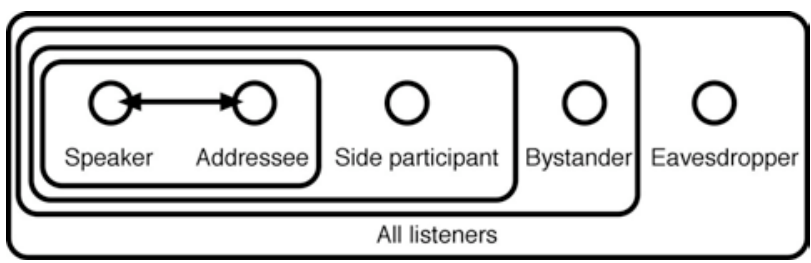

Figure 1. Clark's characterization of participants (1996).

Take, for example, Uncle Roy All Around You (URAY; [5]) which is a street-based performance mixing digital technology and live performance carefully orchestrated to explore the boundaries of public game play. In URAY members of the public volunteer to take part in the performance and are referred to as players. Performers give players instructions which they use with digital technologies to find clues in the public environment to complete their task in the game. Two performers work in specific physical locations such as the main office, three performers move about in the public environment to monitor the players and intervene if necessary, and one further performer operates a central control role. As the game takes place in a public space such as a city, members of the general public who are not part of the game may also become involved by implication.

From this brief description it is clear that there are a set of performers who know, and to some extent construct, the performance frame for the players. There are members of the general public who, as they are not part of the game, are bystanders as they know nothing of the performance frame. In addition there are people who have completed the game and remain in the public space who could be regarded as audience as they understand, to some extent, the performance frame, yet they do not directly take part in the performance. Finally, there are the players who are neither performers (they cannot construct the performance frame), nor spectators (they have some knowledge of the performance frame, and they have a central, interactive, role to the performance). Clearly, a third 
category of behaviour is required - participating - participants are those who interpret the performance frame and carry out action within it.

This is a subtle, but important distinction [cf. 23]. An audience at a football game is an important part of the performance - the cheers and chants spur on the teams and create a sense of occasion in the stadium, but an audience members' primary focus is on interpreting the performers' actions, not directly contributing to the performance itself. When an audience member climbs onto the pitch, they become a participant (albeit unwanted) in the performance - their focus shifts from primarily interpreting the actions of the performers to interacting within the frame.

Benford et al.'s framework does not account for participants in a performance, and moreover, does not account for changes in witting behavior from spectating to participating, and from participating to performing. Transitions between spectating and participating are crucial to the understanding of, and designing for, enticing and engaging interaction - we need to understand how to design performance environments to encourage spectators to wittingly move from being an audience member into actively participating within the performance frame (and back to audience member again!).

To better understand the transitions in wittingness and how to design for them, we first need to characterize what we mean by the behaviors of spectating, participating, and performing. We do so in the next section.

\section{CHARACTERIZING BEHAVIOUR}

In this section we detail our framework for characterizing different forms of witting behavior with respect to a performance frame. As we are concerned with designing exertion interfaces to support transitions in witting behavior our framework focuses on the front of house behavior and does not account for people involved back of house in the orchestration of the performance such as technicians, ticket handlers, etc.

Drawing on Beeman's framework [4], which distinguishes between analytic, technical, and interpretative skills of performers, we characterize behavior with respect to a performance frame in terms of:

- Wittingness: to what extent they know of, understand, and manipulate the performance frame.

- Technical skills: what skills are employed.

- Interpretative skills: how their skills are used to convey meaning.

We first describe the highly witting behavior of performing, then participating, and finally the least witting behavior of spectating which is decomposed into witting audience and unwitting bystanders. Simply put, bystanders do not know that a performance frame exists, an audience is aware of its existence, participants understand and can simply act within the performance frame, and performers can manipulate and perform within the frame to convey meaning.

\subsection{Performing}

Performers are highly witting - they have prior knowledge about what types of actions can affect the interactive system and how these actions are tied to particular effects. Not only are performers aware of these perceived effects but the perceived effects are also visible to witting participants and the audience. Therefore, the performers' concentration is split between multiple interfaces: the interface between performers and the system; performers and participants; and, between performers and audience.

We propose that performing is characterized by the following behaviors:

- Wittingness: Performers understand, and can manipulate the performance frame in which they perform.

- Technical: Performers must develop the necessary motor skills to actually carry out the performative activity. To improve skill, performers must continuously rehearse, a process which Schechner describes as 'twice behaved' [22] or repeated activity. For example, timing is a key technical skill which is needed to ensure that a performer's meaning is conveyed effectively.

- Interpretive: Performers develop a method of making the performative activity uniquely their own - an embodiment of their own skill. Fresh representation of material captures an audiences' attention and allows performers to engage with the audience through charisma, focus and concentration, and freshness and spontaneity. Performers aim to be transformational or 'effective' [4] - they undertake cultural work in the world and aim to leave those involved in the performative act in a changed state. In order to achieve this, performers must be able to focus fully on the performance which relies on their technical skills and effective interaction with systems.

Clearly individual performers will exhibit different skill levels, interpretation, and transformational aims. The key is that evidence of these behaviors indicates highly witting performance in which people enter into a state of flow [10]. Needless to say, performance is not always successful as evidenced by: pushing, or showing obvious effort in the performance which can be distracting; losing concentration; under-preparation; over-preparation which can de-humanize a performance and make it less believable; miscalculation of context which can cause offence; and miscalculation of environment where the potential hazards in the physical environment are misread or neglected (cf. [4])

However, DLA is not simply about how performers interact with the system and audience but also how participants and the audience itself engage with the performance. Taking performing as a start point, we now begin to characterize participating and spectating.

\subsection{Participating}

Participating in a performance involves the acquisition and execution of simple routines rather than extended rehearsal of skills. Participants actively choose to step into the performance frame. However, they do not possess a deep understanding of the performance frame, nor the knowledge or skills to know exactly which actions will have particular effects on the system. Instead, participants acquire skill with the interface through observation of others and trial and error. Usually this activity is accomplished in front of an audience. Participants then, are informed interactors who can acquire and execute simple routines in front of an audience.

We can characterize participants' interaction by the following behaviors:

- Wittingness: When the participant becomes aware of the performance frame they choose to enter into framed behavior. They do not manipulate the performance frame, but, like performers, participants enter the frame knowing that they are being observed by an audience, other participants, and performers. 
- Technical: Participants are able to quickly and easily acquire and execute simple routines to interact with the system and other participants or performers.

- Interpretive: The simplicity of the acquired routines means that participants lack the interpretative skill of performance - they do not attempt to convey meaning through their interaction.

Interactive systems which are too complex for participants to easily execute simple routines cause disruption and disengagement. For example, a system may be too complex for a participant to acquire and execute simple routines within a short time. Similarly, a person may not be willing to interact with a system because the context is misread and is considered esoteric, offensive or inappropriate.

\subsection{Spectating}

In keeping with Benford et al. [5] and Sheridan [24], spectators are divided into two categories: witting audience and unwitting bystanders depending on whether or not they are aware of the performance frame. However, neither audience members nor bystander engage in performative interaction. So while an audience's presence is essential to a performance, their performative behavior is not. We characterize their behavior as follows:

- Wittingness: Audience members are aware of the performance frame, bystanders are not.

- Technical: Spectators do not demonstrate any technical skills with respect to the performance frame.

- Interpretive: As they do not demonstrate any skills, they do not convey meaning.

A bystander is never witting - bystanders witness the performance but are unaware that they are part of the performance frame. They become part of the performance frame by simple implication. This necessarily requires a hidden audience; an informed audience witnesses the performance frame but do not make their awareness known to the bystander. So the audience's presence and behavior is essential to the performance.

When the performance frame is revealed to the unwitting bystander, they then transition to audience member. If they then choose to enter the performance frame and engage in performative interaction, their behaviour changes once again, and they transition to participant.

The distinction between performer and bystander can be a tricky one. If someone is an unwitting bystander and it is the very act of watching him or her that is essential to the performance, then why would they not be characterized as a performer? We look to our characterization of a performer to better understand why we can make this distinction. As stated in our characterization, performers undergo a rehearsal process and are aware of the performance frame. Performers display 'highly informed' interaction. The unwitting bystander has no knowledge of the possible limitations of the system and therefore cannot display any skill.

How useful are these measures in describing witting transitions? We have developed an embedded computing system that we designed specifically to encourage witting transitions from audience member to participant and on to performer. We then used our system in a public performance so that we could interrogate our characterizations. We describe our system and performance in the next sections.

\section{4. iPoi}

We introduced our system iPoi in [3] and discussed not only its capacity to tempt a passive audience into performative interaction but also its ability to intersect with the world of embedded computing. Here we describe the technical limitations of these earlier systems and illustrate our updated wireless, peer-to-peer multi-channel system. First, we provide a short description of poi for those readers who may be unfamiliar with these particular objects.

Poi [16] is an ancient Maori art. Poi are simply balls (poi is a Maori word for ball) attached to chains or cords that are held in each hand and swung around the body in circular movements to create both simple and complex patterns in the air and around the dancer (Figure 2). In Maori culture they were originally constructed with a small rock on the end of a flaxen cord and were traditionally used by men and women to improve flexibility, strength and co-ordination for both work and war. These attributes make poi a particularly interesting object to use as an exertion interface in that they encourage really physical activity on the part of the performer. Today poi are popular objects adorned with tails and ribbons that glow in the dark or can be fixed with tiny flashing lights and are regularly used at clubs and festivals as a means for clubbers, or 'poiers', to gain access to and merge with the driving $4 / 4$ beat of techno and trance music within the dance space. Like juggling, the physics of poi can be attributed to momentum, gravity, centripetal forces and acceleration. Small circular hand movements and wrist rotations are amplified at the end of the cord and thus the momentum and impact is increased creating a dynamic and fluid visual display.

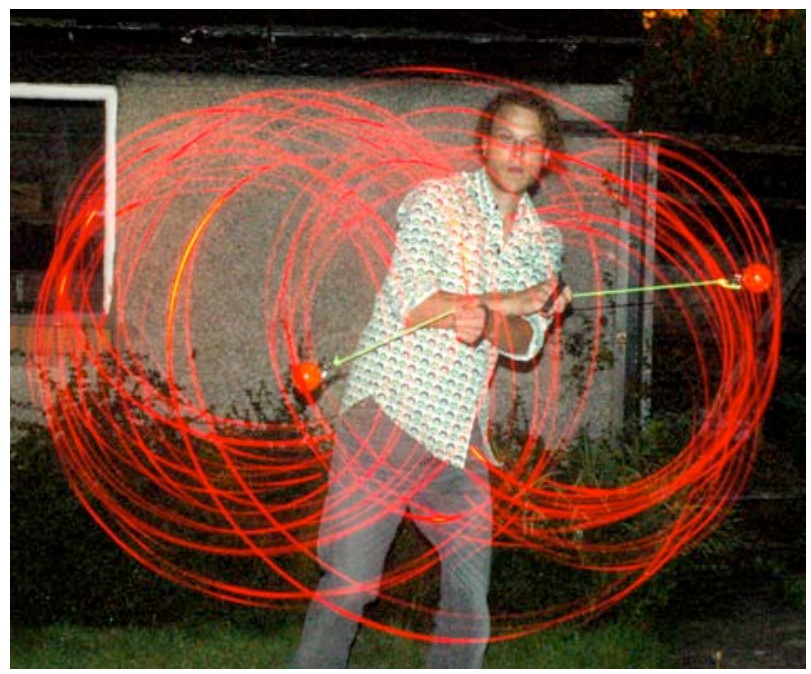

Figure 2. A time-lapsed photograph of an expert poier spinning traditional poi balls showing the 'Butterfly' poi pattern.

By embedding a traditional set of poi with wireless technology we aimed to augment this amplification still further so that the relationship between the performer (the poier), the performed object (the poi) and its performance output (physical, visual and auditory manifestations) was extended and stretched to capacity. As swinging poi is generally a solitary activity and poiers regularly claim they are unaware of people watching them dance, we wanted to investigate how external manifestation of the object's movement through space could influence and increase the desire to interact with others and with the aesthetic environment created as a direct result of the poi's flight. We call our system iPoi. 
iPoi is not a game, but rather a dance with a low entry fee that encourages spontaneous improvisation (Paidia, pure play). Multiple participants improvise their own version of the ancient dance through a demonstration of their traditional poi skills which are then fed back to the audience through realtime visual and sonic output. iPoi has no winners but rather it encourages people to teach, learn and share skills through watching and participating in a performance.

\subsection{Multi-Channel Wireless Prototype}

Our intention with the prototyping phase of the project was to augment poi so as to facilitate witting transitions in performative behaviour. We describe our earlier prototypes in $[3,24,25,26]$ which examined how each prototyped object worked closer to our goal of facilitating witting transitions.

Both our wired and first wireless prototypes certainly encouraged witting performance to some extent. However, the limitations of both of the systems meant that we could only transmit data on a single channel; only one poier and one poi ball could be used at any one time. This limitation meant that the data flow was transmitting from one poi ball only and therefore captured data from one poi in motion (in other words, half of the poi pattern being performed). To detect full poi patterns, we required a system that would allow us to collect data from both poi in real time.

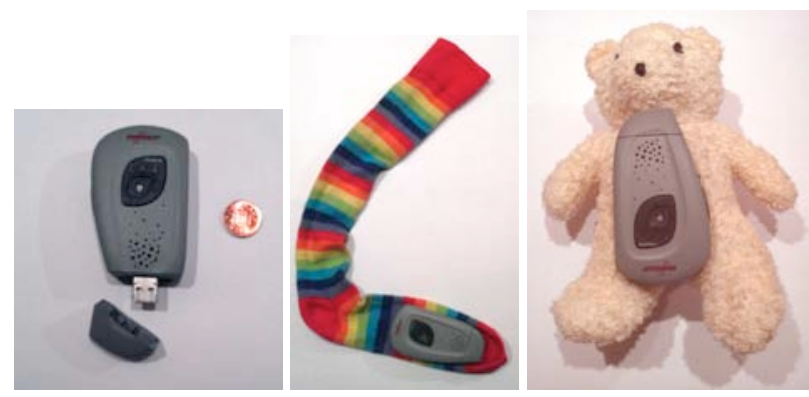

Figure 3. Motes (left) are placed in long socks (middle), and a teddy bear (right).

Our final prototype focused on allowing multiple users to mutually engage [7] with each other using poi to create one communal, dynamic sound and visual piece. Bryan-Kinns et al. [7] define mutual engagement as 'the points at which people spark together, lose themselves in their joint action, and arrive together at a point of co-action'. This meant that we needed to extend our wireless interface to allow for multi-channel data transmission (i.e., transmitting signals from multiple poi simultaneously). The Mote board provided this interface (http://www.moteiv.com). A similar system was used in [17]. It was also at this prototyping phase that we decided to formally name our system iPoi so as to distinguish the system from the more traditional forms of poi.

The Motes themselves are placed in long socks and the poier then holds one sock in each hand and swings them around the body (Figure 3). In addition, a Mote is placed inside a teddy bear (Figure 3) to afford the more 'inconspicuous' action of shaking rather than swinging for shy participants. Data from the Motes are transmitted to a base Mote attached to a PC. The acceleration data are then wirelessly transmitted to another PC where they are used to create visual imagery and audio soundscapes. The system we developed is dynamically reconfigurable allowing us to connect several $i P o i$ and computers on the fly to create ad hoc installations, for example, allowing DJs and VJs to interact with the data from the swinging iPoi (Figure 4).

We demonstrated iPoi at two conferences [25, 26]. The third performance took place at a dance studio as part of a digital cultures festival. We describe this studio performance in detail here as it provided us with a much richer group of performers, participants, context and environment than both of the conferences and was framed as a performance event rather than as a theoretical demonstration.

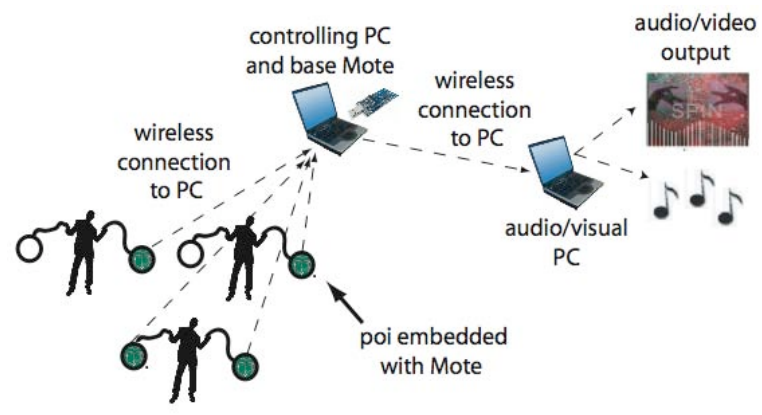

Figure 4. Configuration of wireless prototype with Motes.

\section{PUBLIC PERFORMANCE}

iPoi was the first performance in a two-week festival which was open to the public. The public was invited to the event through the center's website, email invitations and pamphlets distributed through the center's mailing list. A steady stream of people attended the two-hour evening event, coming and going as they pleased. Given the public nature of the event we can only estimate the number of participants involved based on our observations and post-hoc video analysis. We estimate that approximately 75 people participated. It is important to note that performances involving poi often draw an audience of expert poiers. However, we did not explicitly invite any poiers apart from the two whom performed at the beginning of the evening.

\subsection{Procedure}

Our position is that we needed to look beyond conventional empirical research methods to understand transitions in wittingness that occur in DLA. This is because the nature of interaction that we are interested in is typically not repeatable experiments in controlled environments but rather public performance. In public performance whilst the technology may stay the same, the context, participants and observers change between events. Likewise, the motivation for participating in empirical experiments is different to the motivation to attend public performances. For example, participants in lab-based experiments are often rewarded through payment whereas participants (or audience) in public performance typically pay to watch a performance and attend as a social activity.

Here we outline the procedures we employed in an attempt to gather observational data. Our performance required several people who were responsible for different tasks. Before the performance began, invigilators were briefed as to how the system worked so that they could answer inquiries from the audience. This was particularly important as we did not provide any signage about how the system worked. One person was responsible for monitoring the audio system as well as the realtime streaming of the acceleration data. Another person was responsible for monitoring the visual output as well as video recording the performance with one static camera and one 
roaming camera. Two performers were responsible for designing an orchestrated performance.

Prior to the performance, the performers had about one hour in which to rehearse their performance. During this time, the performers practiced their traditional poi skills individually with the $i P o i$ and collaboratively. Both of the performers were experienced poiers (one expert and one intermediate) who had rehearsed with $i P o i$ several times before the evening but had not performed together.

During the rehearsal the two performers identified the strategies they needed in order to entice witting participation and discussed these strategies with the investigators. This included: where to place the iPoi to make them accessible to the audience; the audio sequence they preferred; who would perform first and for how long; when they would perform together; and, which poi patterns they would perform. Together they identified how they could 'build up' the performance into a layered narrative with a clear climax happening just before they handed over the iPoi to the audience. The performers displayed their skills to each other and identified which poi patterns they wanted to perform.

Additionally, the two performers intentionally wore simple clothing - a t-shirt and jeans to show that the technology did not rely on someone wearing special clothing to interact with the iPoi.

During the performance, the investigators approached participants and spectators and asked them informal questions about how their experiences in interacting with iPoi. A few days after the performance, the investigators interviewed the two initial performers and recorded their observations on paper.

\subsection{Technical Set-Up}

The installation had three basic components: sets of poi embedded with Motes, three computers, and projector facilities. The event took place in a location which usually functioned as a dance studio - a large rectangular room with tall windows down one length of the room and at one end, mirrors along one end and a balcony (Figure 5). As there was no method of attaching a projector to the ceiling, we had to fix our projector on a large stepladder in the middle of the room which was covered with black cloth. (This in itself became an unintentional but rather significant object in the room, the effect of which will be discussed later.)

From our earlier guerilla performance, we recognized that not everyone is comfortable performing in front of groups of people and that sometimes the expert poier made the system look difficult to use. To address this issue, we secreted a Mote inside a small teddy bear (Figure 3). The teddy bear afforded the action of shaking, a subtler movement and a rather more inconspicuous object than the $i P o i$.

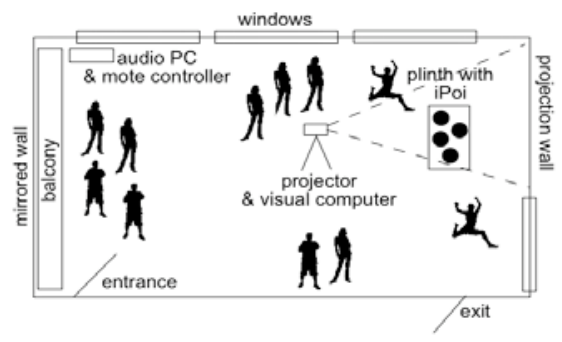

Figure 5. iPoi set up at the dance studio.
We used three computers to control the audio and visual elements. One computer collected the realtime data wirelessly from the Motes. These data were then sent wirelessly to the audio application running on another computer. Both of these computers sat in the far corner of the room underneath the balcony in a metal box. Our third computer sat underneath the projector on the ladder in the middle of the room. This computer, which ran our visual output, was connected to the projector. The projector then cast the resultant image onto a white wall. To create a more defined performance space within what was otherwise an empty room with no lighting to direct focus or delineate particular areas, we placed several iPoi on a plinth between the projector and the projection wall.

Each $i P o i$ had the capacity to trigger one set of pre-recorded audio samples and one pre-made visual. So swinging one iPoi would contribute to both a soundscape and an image layered over the top of the background image and sound. Swinging several iPoi would create additional layers in much the same way as a DJ or VJ might layer tracks and images over each other to create an individual interpretation of the raw materials available. For this particular installation one iPoi controlled a digital animation of a shadow puppet dog on the left hand side of the screen. The longer a performer swung the iPoi, the further the dog would move towards the middle of the projection screen (Figure 6). A different iPoi controlled a similar dog on the right hand side, another controlled random words and a fourth created a real time plot of acceleration data on the bottom of the screen. (Important to note is that the sounds and visuals did not cancel each other out - images and visuals were calibrated so that they complemented each other to create one coherent layered soundscape and visual projection.)

Two soundscapes (referred to as $f x$ and $\operatorname{dog}$ music) were created for the piece and played using Ableton Live (http://www.ableton.com). Each consisted of a continuously playing loop of five structured musical tracks which were selectively activated by the objects when they moved, and one track which continuously played a simple heartbeat. Both soundscapes had a dance/techno feel and played at $120 \mathrm{bpm}$ (although $f x$ had a softer, more organic quality than dog music). The audio soundscapes were changed half way through the evening so that the performance stayed engaging and fresh.

\subsection{Method}

Prior to the performance, the doors to the room were shut so that people gathered outside. People entered the room in one group, and stood where they wished. A default projection (red bubbles) played on the screen and single sound (a heartbeat) played on the speakers. Two witting performers stood within the audience. When the crowd had settled in, we began an orchestrated performance in an attempt to entice various transitions in witting behavior. Since we expected that many people were unfamiliar with both the art form and the technology, this introductory performance acted as both a demonstration of poi as a dance and display of some physical dexterity and as a demonstration of the augmented technology. In this initial performance, observers were given visual and audio clues as to how they could interact with the system. In this way we aimed to help the audience move from spectating to participating.

One performer emerged from the crowd and walked up to the plinth. She took one iPoi in her hand and stood silent for a moment. She then swung the $i P o i$ around her body slowly in a circle at first, and then gradually increased the action with speed and changing patterns. Whilst doing so a new image and sound filled the projection and speakers. Occasionally she 
would stop spinning, which would cause the sound and image to disappear. Eventually she picked up another $i P o i$ and swung both of them around her body, again creating new layers of sounds and images. She continued performing for several minutes as the audience stood back and watched. After some time, she placed the iPoi back on the plinth and walked back into the crowd. When she did this, a second performer emerged from the crowd and repeated this performance but with more intensity and with a different range of established poi patterns. After several minutes, the first performer returned and picked up two iPoi. The two performers swung iPoi around their bodies and together they created layered visuals and audio soundscapes with the intention of bringing to the audience's attention the correlation between the objects moving in space and the resulting change in the digitally mediated environment. After the initial performance, the two performers walked into the audience and offered the iPoi to people in the crowd.

Upon giving the crowd the iPoi, we were interested in noting the transitions from spectating to participating, and from participating to performing. Next we explore the effect our prototype had on these transitions.

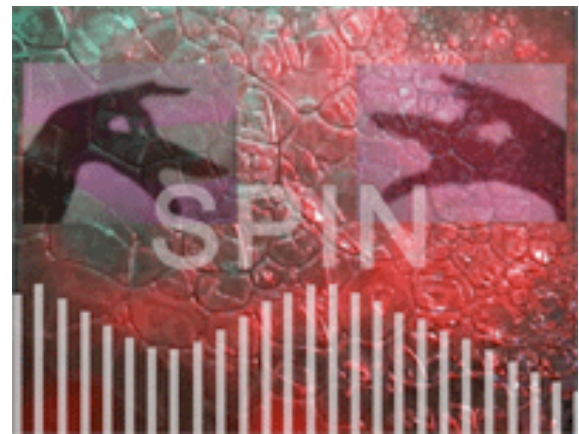

Figure 6. Layered projected image created when spinning several $i P o i$ simultaneously.

\section{OBSERVATIONS}

In reflecting on our characterizations of witting behavior, we can say that in this particular performance, everyone was witting to some extent. People entered the performance event with some knowledge about the performance frame - they knew that they had come to a room to experience some sort of performance. So in this case, we do not have any bystanders (unwitting spectators) - everyone who entered the room was at least a witting spectator (i.e., a member of the audience).

\subsection{Spectating}

Although many people transitioned to participating and performing, there were some people who were happy to simply watch the performance. Others, however, refused to interact with iPoi even when invited to do so by others. For those who did not 'get it', even though they tried to have a go with the objects and acquire the necessary skills, the mapping from object to audio and visuals was clearly inadequate to allow them to transition to participation, and so they remained as witting spectators - they knew what was going on, but not how to acquire the skills to join in.

For those who remained witting but unwilling spectators and abstained from interaction altogether, other issues may have come into play such as the fear of making a fool of oneself in public. As with many public performances which attempt to involve the audience, it is clear that not all people who attend will want to become full participants in the action; our intention as designers is to facilitate the transition from spectator to participant but to respect people's right to remain in the spectator role and to acknowledge its significance in maintaining the core of what it is to engage in live performance where people observe others at some physical and psychical distance.

\subsection{Participating}

Our aim was to create a playful environment in which people could slide easily from spectating to participating. Simply moving the $i P o i$ or shaking the bear caused sound and visuals to be produced giving immediate feedback which we hoped would allow people to quickly and easily acquire and execute simple routines through experimentation.

Right after the initial performance a few witting spectators took the $i P o i$, walked in front of the projection screen and began swinging them. In other words, they entered the performance frame - they became participants. They continued swinging and spinning them until someone else came up and asked to try it out or until they passed it on to one of their friends or someone else in the audience. This passing (an example of social infection [24] facilitated by the simple interactive nature of the iPoi) sometimes included a short discussion on how to use the object, but more often than not people just gave the objects a go to see what happened.

Note that people's descriptions of how to interact with iPoi were all self-constructed narratives as we had not provided any explanation. Certainly these self-constructed narratives increased levels of interaction and engagement within the room and became an interesting facet of the event as theories, ideas and reactions to the performance filtered amongst the participants both verbally and through the embodied movement facilitated by the objects themselves.

Through observation, experimentation, and constructed narratives many participants were able to acquire and execute simple routines with iPoi in a short amount of time - evidence of participating. Several people spent a significant amount of time swinging $i P o i$, trying various patterns, and swapping different $i P o i$ with other people. As they did, they transitioned from witting spectators to participants who had an understanding of the effect of their action within the performance frame.

A few expert poiers began swinging the iPoi as they normally would spin traditional poi. We see this as using previously learnt skills to help transition from witting spectator to participant. These participants then paid attention to the effect they were able to have on both the sounds and images generated by the system, and those spinning poi near them. In this way they honed their skills and may have started to perform.

In terms of design, it seemed that it was easier for participants to relate their movements to the sound produced rather than the video. From this point of view it was through understanding the audio interaction that participants were able to transition to participation. Interestingly, this understanding was usually gained by stopping the movement of an object. Indeed, we observed a pattern of people who were transitioning to participation moving the objects, being unsure what was happening, stopping the movement, concentrating on listening for what was missing from the audio mix, looking at the screen, and repeating until they 'got it', and in doing so acquired the necessary skills to participate. After a few stops and starts people would typically let out a short 'ah-ha' expression and then use the object without such focused attention to what they did, but with more appreciation of how they may be contributing to the performance. Becoming participants at this 
point they then started on their journey towards transitioning to become performers in the space.

Whilst many people transitioned to participation, it was clear that they had some difficulty in determining what audio they were affecting. Indeed, when all tracks were playing it was very difficult for participants to understand what was going on in general, let alone what they had control of. This was probably due to the large number of audio tracks that could be playing at any one time, the changing structure of the tracks, and the similarity of some of the prerecorded music. We see this problem as hindering a transition from participation to performance - whilst participants were aware that they were doing something they were not able to express themselves as much as we had hoped. Specifically, the somewhat opaque mapping from object to sound and visual hindered their technical skill acquisition and rehearsal. This in turn hindered the complete understanding of the performance frame, and prevented participants from developing a method for making the activity uniquely their own and expressing themselves fully. We intend to further explore these issues in future developments and performances.

\subsection{Performing}

Despite the limitations described above, several participants did begin to structure their performance with respect to the soundscape and to each other which indicated to us that they had transitioned from participating to performing. Evidence of this transition is especially important for the design of DLA as it allows people to become performers without extensive rehearsal and skill acquisition beforehand.

Two key forms of behavioral structure emerged which indicated to us that people were performing. We saw this as evidence of deep understanding of the performance frame, advanced technical skill, and attempts at self-expression. We classified these structures as: trading pauses and emphasizing beats. In trading pauses performers interacted with each other by purposely pausing one of their tracks whilst the other played, and vice-versa. Anecdotally, this had a similar structure to the convention of trading licks in jazz improvisations, but utilized the ability to turn tracks on and off to create the opposite effect. More experienced poiers who used $i P o i$ for an extended period of time developed this trading of pauses to encompass four tracks which generated a performance with significant auditory depth. This required significant technical skill in order to stop tracks playing at appropriate points in time. Specifically, performers had to time their spin so that they could stop the iPoi at the bottom point of the spin at a time which would fit with the other iPoi they were swinging, and the person they were trading pauses with. It also required deep understanding of the performance frame in terms of understanding the tolerance of the system for movement in the iPoi (i.e. when the system would decide to pause the sound), and the reaction time of the system (to ensure that the pauses were in time with the music and others' actions).

The second form of structure involved performers emphasizing beats by playing their track only for one beat (for example, at the start of a bar). This structure was seen in individual interaction and as ensemble interaction where the aim was to emphasize beats of the other participant, or to jointly emphasize beats in the soundscape itself. The development of these structures by performers relied on eye contact, adherence to the beat of the soundscape, and very little verbal communication. As such it illustrated the expressive power of an environment with very simplistic but intuitive and naturalistic control mechanisms. As with trading pauses, this form of performative action relied heavily on deep understanding the performance frame, and high levels of technical skill in order to co-ordinate the sounds. Moreover, the layering of music was used by performers to create musical tension and release which engaged the audience, participants, and performers, and moreover, conveyed emotion.

It is worth noting that as the speed of iPoi swinging had no influence on the speed of audio, the poiers tended to spin their $i P o i$ in time with the music. This reinforces the idea that performers were not simply operating the $i P o i$ in isolation, but were part of the collective experience - attuned to the speed of music and each other's iPoi swinging.

\section{DISCUSSION}

Our investigations with iPoi led us to consider a range of practical and pragmatic considerations when developing technologically-mediated works which play with the witting, and the facilitation of movement between spectating, participating and performing. Prototyping several objects and testing them in real-world environments has raised a number of issues about how to design technological objects that encourage witting transitions in the performative mode. We consider some of these issues here.

\subsection{Orchestrated Performance}

Having two performers perform with iPoi in front of the audience through an orchestrated performance (albeit an informal one) proved critical to encouraging witting transitions. The performance set up the frame and allowed the audience to understand the possibilities and limitations iPoi in a distanced and low-risk manner. Not only did the orchestrated performance show off the technical and interpretive skills of the performers, but also served as performative modeling for the audience, giving them clues as to how they might enter the performance frame and engage with iPoi themselves. This orchestration functioned as a rapport-building exercise at the start of the evening where the audience was invited into the action in the same way guests may be drawn into a conversation at a party. The performers acted as hosts and handed over the performance tools (objects, space and context) to the audience so that they could make it their own.

\subsection{Learning to Interact}

People learnt how the iPoi worked by observing each other interacting with them, through their own encounters with the objects, and by word of mouth, or 'social infection' [24]. Learning through observation and word of mouth changes someone's wittingness - they develop greater understanding of what is possible within the performance frame. Learning through interacting with the objects themselves increases both wittingness and technical skill as they acquire and rehearse simple routines.

There are two design factors that are critical if we want to create objects which we can learn to use through observation, word of mouth and experimentation: observable and intuitive interaction. We suggest that the basic mode of interaction (swinging) with iPoi is easily observable to a room full of people. Firstly, iPoi are swung around a body and they have a physical range of interaction of about $1 \mathrm{~m}$ from the hand. This is significantly different to interacting with, say, a mouse, a PDA, or even a touch screen which may have a physical interaction range of around $40 \mathrm{~cm}$, and makes iPoi interaction easily observable. Secondly, the swinging of the iPoi means that interaction is observable from all around the poier, not just by looking at a fixed point such as a touch screen which may be 
obscured by the performer and other people in the space. Again, this increases the observability of the interaction.

\subsection{Some People Didn't 'Get it'}

Some people were reluctant to interact with iPoi regardless of the encouragement they received from others. These unwilling audience members said that they didn't understand what was happening and thus felt compelled not to accept the invitation to participate. Whether they felt embarrassed or intimidated is an issue worth further consideration. We observed that those who did not 'get it' (including some witting participants) had difficulty understanding the mapping between swinging/ shaking and to visual and audio output, particularly when there were multiple people involved. They were witting as they knew that swinging the iPoi has something to do with the performance, but they did not know what the swinging caused to happen, nor why.

In future development, we intend to make the mapping between iPoi and audio and visuals clearer. For instance, we could use color to make an explicit connection between different $i P o i$ and their associated visuals - e.g. a yellow iPoi would produce a yellow visual, and a red $i P o i$ would produce a red visual. We are also interested in mapping between the pattern of interaction and the visualizations. For example, swinging an $i P o i$ in a circle could create a circle on the screen which might make the mapping more intuitive and observable. Since the patterns being produced change extremely quickly, collecting the data and recognizing the patterns in real time is a difficult task indeed.

\subsection{Physicality}

In keeping with Winkler [29] we found a strong relationship between the physicality of the object and people's intuitive expectations of the sound it produced. This was most clearly illustrated with the bear which produced a jangly guitar riff in the $f x$ soundscape, and an offbeat percussive track in dog music. Both tracks complimented the soundscape in key and rhythm, but the jangly guitar riff of $f x$ was significantly easier for participants to relate to the bear than the percussive track. For us this meant that it was harder for audience members to understand the teddy bear's relation to the performance frame when the dog music soundcape was used. There may be several reasons for this as outlined below.

Firstly, the guitar riff was significantly sonically different to the other tracks in $f x$, whereas there was another percussive track in dog music. As such, the guitar riff could be identified in the mix easier by participants, that is, its role in the performance frame was more observable. Indeed, the percussive track in $\operatorname{dog}$ music needed to be significantly louder in the mix than other tracks to make it noticeable to participants.

Secondly, we suggest that the weight, size, shape, softness, and furriness of the bear affords a certain style of interaction usually a soft shaking akin to churning or waggling. The waggling action fitted with the sonic quality of the guitar riff which was soft and warm in the mix, whereas the percussive track of dog music was hard, and jarring. So, participants perceived a connection between waggling the bear and the guitar riff which played at a similar tempo.

Similarly, there seemed to be a clearer relationship between the iPoi and tracks which made continuous sound and which had some sort of flange or chorus effect. The continuity of the sound and the effect applied to it seemed to relate to the swinging of the iPoi better than percussive beats. In previous iterations we found that a mote on its own seemed to be related well to percussive tracks which we propose is due to its small size and hard, technical, angular appearance which people tended to interact with by shaking vigorously, much as one would an egg-shaker percussive instrument.

We take from this that the relationships between the physicality of the object, the forms of interaction it affords, and the sound and vision it controls need to be carefully designed to allow people to understand how the object fits into the performance frame. Current research on tangible interaction such as Hornecker and Buur [14] and Williams [28] tends to overlook the continuous nature of interaction with objects such as iPoi where it is the constant movement of the object that is the focus of interaction rather than its physical position or orientation. Notable exceptions tend to be in the music domain (see [29] for a survey) and include systems such as the Radio Baton which allows people to 'conduct' a piece of music in real time by continually moving an augmented baton.

\subsection{Virtuosity}

Having several expert poiers in the audience contributed significantly to the success of our performance. They were the first ones who wanted to participate once the performance was opened to the audience and the last ones to leave. One expert poier interacted with $i P o i$ for the entire performance and he was keen to show people how it worked and to interact with them in the performance space.

However, we do feel that the current design of iPoi limited the technical skill that performers could develop. In particular, the limited control of the audio soon became apparent during interaction, especially with experienced poiers. There were expectations that swinging iPoi in different patterns, at different speeds, or with different strength would somehow change the audio. Typically performers expected that swinging the poi quickly would increase the volume or speed of their track. Generally, these were people who were already familiar with poi in its traditional form and so were able to use some of their previously rehearsed technical skills with $i P o i$. We are currently working on means to allow greater interaction with the visuals and audio which we hope will allow performers greater interpretative scope in their performances with iPoi.

\subsection{Inconspicuous Interfacing}

Some audience members seemed somewhat intimidated by participants who clearly exhibited expert technical poi skills. We observed that people who were reluctant to swing $i P o i$ were much more willing to interact with the teddy bear. The physicality of iPoi (swinging a long sock) meant that participants stood out in the crowd - they had to move to a space in which they could safely swing the object around without hitting others. However, the physicality teddy bear (shaking up and down), meant that they didn't have to move from where they were standing and could remain hidden in the crowd. This suggests a need to design exertion interfaces for those who want interact in a more inconspicuous manner.

It is interesting to note that the teddy bear anecdotally required more word of mouth explanation than the iPoi. There are several possible explanations for this including the fact that interaction with the teddy bear is less observable than with iPoi as it is held within the hand and shaken relatively close to the body in comparison to the iPoi. Another factor may be that the teddy bear was used by people who were uncomfortable with interacting with the iPoi as they had less understanding of the performance frame and the possibilities for interaction which meant that interaction had to be explicitly outlined to them. 


\subsection{Non-Stage and Breaking the 'Mystery'}

Having a projector on a ladder in the middle of the room caused several problems. Firstly, it broke the 'mystery' of the technology since many people assumed that there was something hidden under the ladder which was controlling the visuals and soundscapes. Because the ladder was so big, it obstructed the view for the audience and split the room into two spaces - a 'performance space' and an 'audience space'. We did not want a 'stage' but rather to encourage people to move about the room and to perform anywhere. However, the large ladder prevented this from happening. In future performances, we will provide detailed diagrams and a video of the performance so that the curators understand the technical requirements.

\section{CONCLUSIONS}

We presented a framework for characterizing different kinds of behavior with respect to a performance frame. This was used to illustrate how people transitioned from spectator to participant and on to performer with our DLA, iPoi, and draw out observations on the nature of designing for performance. We are currently developing iPoi to make it easier to enter into the performance frame, and to provide greater scope for technical skill development. Also, we are currently assessing the technical components needed to further our understanding of how to create visualizations and sonifications with realtime acceleration. As well, this work is applicable to a wide range of applications and participant groups beyond the scope of this paper - for example, challenged and young participants. We intend to create specific guidelines which can be applied to various applications and domains. We will explore the applicability of our framework to these groups and applications as well as other DLA and domains.

\section{ACKNOWLEDGMENTS}

We would like to thank Matt Cooper for his awesome poi skills and project assistance. This work is partly support by the EPSRC Equator IRC (GR/N15986/01), LeonardoNet Network Grant (GR/T21042/01), and the EPSRC Engaging Collaborations Grant (GR/S81414/01).

\section{REFERENCES}

[1] Anderson, K. 'ensemble': Playing with sensors and sound. In CHI 2004: Extended abstracts of the SIGCHI conference on Human factors in computing systems. ACM Press, New York, NY, 2004, 1239-1242.

[2] Bateson, G. A theory of play and fantasy. Psychiatric Research Reports, 2 (1955), 39-51.

[3] Bayliss, A., Sheridan, J. G., and Villar, N. New shapes on the dancefloor: Influencing ambient sound and vision with computationally-augmented poi. International Journal of Performance Arts and Digital Media, 1, 1 (2005), 67-82.

[4] Beeman, W. O. Performance Theory in an Anthropology Program. [Last checked April 1, 2007]. Available at http://www.brown.edu/Departments/Anthropology/publica tions/PerformanceTheory.htm

[5] Benford, S., Crabtree, A., Reeves, S., Flintham, M., Drozd, A., Sheridan, J. G., and Dix, A. The frame of the game: Blurring the boundary between fiction and reality in mobile experiences. In Proceedings of the SIGCHI conference on Human factors in computing systems (CHI 2006). ACM Press, New York, NY, 2006, 427-436.
[6] Blythe, M. A., Monk. A. F., Overbeeke, K., and Wright, P. C. Funology from Usability to Enjoyment. Kluwer Academic Publishers, London, UK, 2003.

[7] Bryan-Kinns, N., Healey, P. G. T., and Leach, J. Exploring mutual engagement in creative collaborations. In Proceedings of Creativity and Cognition 2007. (Washington, DC, June 2007). 2007.

[8] Caillois, R. Man, Play and Games (Les Jeux et les Homes) (M. Barash, Trans.). University of Illinois Press, Chicago, IL, 2001. (Original work published 1958).

[9] Clark, H. H. Using Language. Cambridge University Press, Cambridge, 1996.

[10] Csikszentmihalyi, M. Beyond Boredom and Anxiety: The Experience of Play in Work and Games. Jossey-Bass, San Francisco, CA, 1974.

[11] Dix, A., Sheridan, J. G., Reeves, S., Benford, S., and O'Malley, C. Formalising performative interaction. In Proceedings of the 12th DSVIS 2005. (Newcastle, UK, July, 2005). Springer Lecture Notes in Computer Science, London, 2006, 15-25.

[12] Fujiki, Y., Kazakos, K., Puri, C., Starren, J., Pavlidis, I., and J. Levine. NEAT-o-Games: Ubiquitous computing meets exertion interfaces. In Proceedings of the 2007 ACM Workshop on Exertion Interfaces. (San Jose, CA, April, 2007). 2007.

[13] Goffman, E. Frame Analysis. Harvard University Press, Cambridge, MA, 1974.

[14] Hornecker, E., and Buur, J. Getting a grip on tangible interaction: A framework on physical space and social interaction. In Proceedings of the SIGCHI conference on Human factors in computing systems (CHI 2006). ACM Press, New York, NY, 2006, 437-446.

[15] Hoysniemi, J. International survey on Dance Dance Revolution game. Computing Entertainment, 4, 28 (2006).

[16] Huata, N. The Rhythm and Life of Poi. Harper Collins, Auckland, New Zealand, 2000.

[17] Kabisch, E., Williams, A., and Dourish, P. Symbolic objects in a networked gestural sound interface. In Proceedings of the SIGCHI conference on Human factors in computing systems (CHI 2005). ACM Press, New York, NY, 2005, 1513-1526.

[18] McCarthy, J., and Wright, P. Technology as Experience. MIT Press, Cambridge, MA, 2004.

[19] Mueller, F., Agamanolis, S., and Picard, R. Exertion interfaces for sports over a distance. In Proceedings of UIST 2002. (Paris, France, October, 2002). 2002.

[20] Mueller, F., Agamanolis, S., and Picard, R. Breakout for two: An example of an exertion interface for sports over a distance. In Adjunct Proceeding of UbiComp 2003. (Seattle, October, 2003). 2003.

[21] Reeves, S., Benford, S., O'Malley, C., and Fraser, M. Designing the spectator experience. In Proceedings of the SIGCHI conference on Human factors in computing systems (CHI 2005). ACM Press, New York, NY, 2005, 741-750.

[22] Schechner, R. Performance Theory. Routledge, New York, 1988.

[23] Sheridan, J. G., Dix, A., Bayliss, A., and Lock, S. Understanding interaction in ubiquitous guerrilla performances in playful arenas. In People and Computers 
XVIII (Proceedings of HCI 2004). Springer-Verlag, London, 2004, 3-17.

[24] Sheridan, J. G. Digital Live Art: Mediating Wittingness in Playful Arenas. Ph.D. Thesis, University of Lancaster, 2006.

[25] Sheridan, J. G., Bayliss, A., and Bryan-Kinns, N. iPOI. In Proceedings of the first international symposium on Culture, creativity and interaction design. (Queen Mary, University of London, UK, 12 September, 2006). 2006.

[26] Sheridan, J., Bayliss, A., and Bryan-Kinns, N. iPoi: Acceleration as a medium for digital live art.

Demonstration. In Proceedings of UbiComp 2006. (Orange County, CA, September, 2006). 2006.
[27] Wessel, D., and Wright, M. Problems and prospects for intimate musical control of computers. In Proceedings of NIME Workshop (CHI 2001). (Seattle, WA, March, 2001). 2001.

[28] Williams, A., Kabisch, E., and Dourish, P. From interaction to participation: Configuring space through embodied interaction. In Proceedings of UbiComp 2005. (Tokyo, Japan, September, 2005), 287-304.

[29] Winkler, T. Making motion musical: Gesture mapping strategies for interactive computer music. In Proceedings of the 1995 international Computer music conference. (Banff, Canada, September 3-7, 1995). 1995. 